\title{
Qualidade de Vida de Professores do Ensino Fundamental de uma Escola da Rede Pública
}

\section{QUALITY OF LIFE OF TEACHERS FROM A PUBLIC BASIC SCHOOL}

\section{Anne Samilly Gomes Moreira', Thayla Amorim Santino ${ }^{2}$, Alecsandra Ferreira Tomaz ${ }^{3}$}

1. Fisioterapeuta graduada pela Universidade Estadual da Paraiba (UEPB), Campina Grande, PB, Brasil.

2. Mestranda em Fisioterapia do Centro de Ciências da Saúde, Universidade Federal do Rio Grande do Norte (UFRN), Natal, RN, Brasil. Fisioterapeuta graduada pela Universidade Estadual da Paraiba (UEPB), Campina Grande, PB, Brasil.

3. Doutoranda em Engenharia de Processos, Universidade Federal de Campina Grande (UFCG). Doutorado em Medicina do Esporte, Universidad Catolica Nuestra Senora de La Asuncion, Paraguai. Professora do Departamento de Fisioterapia, Universidade Estadual da Paraiba (UEPB), Campina Grande, PB, Brasil.

\section{RESUMO}

Este estudo busca avaliar a qualidade de vida dos professores do ensino fundamental de uma escola da rede pública brasileira. Tratase de um estudo transversal realizado com 26 professores. Utilizouse um questionário sociodemográfico, o SF-36, Questionário Nórdico e o Inventário de Depressão de Beck. Observou-se a prevalência do sexo feminino $(69,6 \%)$ e com cônjuge $(60,9 \%)$. A maioria possuía apenas graduação $(56,5 \%)$. Considerando a dor musculoesquelética, foi encontrada maior ocorrência nos membros inferiores $(34,8 \%)$ e na coluna $(47,8 \%)$, sendo que apenas $34,8 \%$ dos docentes entrevistados relataram algum tipo de afastamento por queixa de dor em algum dos segmentos. Apenas 5,35\% dos professores apresentaram algum indicativo de depressão. Quanto à qualidade de vida, constatou-se um escore mínimo de 56,04 para "Estado geral de saúde" e o máximo de 84,38 para o domínio "Capacidade funcional", sendo o domínio "Limitações por aspectos físicos" o mais afetado quando comparado dor com diminuição da qualidade de vida. Houve uma depreciação da qualidade de vida dos docentes que relataram dores, principalmente nas costas, nos últimos 12 meses. Constata-se haver comprometimento de todos os domínios do SF-36, portanto infere-se que a sintomatologia dolorosa e depressiva contribui para uma redução da qualidade de vida do docente.

(Gomes A, Amorim T, Ferreira A, 2017. Qualidade de Vida de Professores do Ensino Fundamental de uma Escola da Rede Pública. Cienc Trab. Ene-Abr; 19 [58]: 20-25).

Palavras-chave: QUALIDADE DE VIDA; DOCENTES; SAÚDE DO TRABALHADOR; EDUCAÇÃO.

\section{ABSTRACT}

This study aims to evalue the quality of life of teachers of a Brazilian public basic school. This is a cross-sectional study done with 26 teachers. It was used a social-demographic questionnaire, the SF36, the Nordic Questionnaire and the Inventory of Beck Depression. It was observed that most teachers are female $(69,6 \%)$ and married $(60,9 \%)$. Most of them have only graduation (56,5\%). Considering the musculoskeletal pain, it was found higher occurrence in lower members $(34,8 \%)$ and in column $(47,8 \%)$, and only $34,8 \%$ of them related some kind of sick leave because of pain in some of the segments. The study showed that 5,35\% of the teachers have an indicative of depression. About quality of life, it was detected a minimum score of 56,04 for "general state of health" and the maximum of 84,38 for the domain "functional capacity", with the domain "limitations for physical aspects" being the most affected when compared pain with loss of quality of life. It was observed a quality of life depreciation on the teachers that reported pain, most of them in their backs, through the last 12 months. All the domains of SF-36 were engaged, then we can infer that the painful and depressive symptomatology contributes to a reduction of teachers' quality of life.

Key words: QUALITY OF LIFE; TEACHERS; OCCUPATIONAL HEALTH; EDUCATION.

\section{Correspondência / Correspondence:}

\section{Alecsandra Ferreira Tomaz}

Centro de Ciências Biológicas e da Saúde, Departamento de Fisioterapia, Universidade Estadual da Paraiba

Av. das Baraúnas, 351, Campus Universitário, Bodocongó

Telefone: +55 (83) 3315-3346

e-mail: alecsandratomaz@hotmail.com

Recibido: 30 de Enero de 2017 / Aceptado 08 de Marzo de 2017

\section{INTRODUÇÃO}

Segundo o Instituto Nacional de Estudo e Pesquisas Educacionais Anísio Teixeira ${ }^{1}$, o Brasil possui cerca de dois milhões de professores na educação básica. Sabe-se que os professores compõem uma das categorias profissionais que mais tem sofrido mudanças devido a intensificação do ritmo de trabalho, sendo ainda uma das que mais sofrem com o estresse e outras sindromes decorrentes do seu trabalho. ${ }^{2,3}$ De acordo com Francelino ${ }^{4}$, "o professor se vê submetido às mesmas condições dos trabalhadores fabris, pois a escola adquire a nova função de formar trabalhadores".

De acordo com a Classificação Brasileira de 0cupações (CBO) ${ }^{5}$, estes profissionais possuem competências especificas, relacionadas 
ao ato de preparar e ministrar aulas, participar na elaboração do projeto pedagógico e ainda planejar o curso de acordo com as diretrizes educacionais. Além destas, o professor encontra-se envolvido em reuniões administrativas e pedagógicas, com a organização de eventos e atividades sociais, culturais e pedagógicas, que objetivam investigar, orientar alunos e interagir com os pais e/ou responsáveis de seus alunos. 0 professor do ensino fundamental ainda deve participar de seminários e de reuniões do conselho de classe, preencher relatórios relativos às dificuldades de aprendizagem e, muitas vezes, cuidar do patrimônio, materiais, recreios e locais de refeições. ${ }^{6}$

Diante de todas as atividades anteriormente citadas, é possivel observar que o trabalho realizado pelo docente do ensino fundamental torna-se exaustivo devido ao contato maior que deve haver com o aluno, diferentemente das outras séries. Somando-se a particularidades como trabalho repetitivo, insatisfação no desempenho das atividades, desgaste nas relações professor-aluno, ambiente intranquilo, falta de autonomia no planejamento das atividades, ritmo acelerado de trabalho, execução das atividades sem materiais e equipamentos adequados e salas inadequadas. Tais aspectos podem resultar em um quadro crônico de depreciação e desqualificação social, psicológica e biológica desses professores, podendo gerar esforço adicional dos docentes na realização de suas tarefas. ${ }^{7-11}$

Vários fatores ligados à organização do trabalho destes profissionais colaboram para o agravo da saúde, pelo fato dessas estarem ligadas diretamente à sobrecarga de trabalho, baixa remuneração e a crescente violência na escola, acarreta em sentimentos negativos com relação a atividade exercida. Este trabalho em excesso e pouco remunerado, adicionado às características individuais do estilo de vida e das condições de trabalho, pode favorecer o surgimento de sintomas osteomusculares, o que tem sido uma preocupação para alguns pesquisadores, pois se trata de uma questão de saúde e trabalho, em virtude dos custos e o impacto na qualidade de vida dos docentes. ${ }^{12-14}$

Diversos fatores sociodemográficos, psicossociais, físicos e organizacionais estão relacionados ao desencadeamento, desenvolvimento e manutenção da dor musculoesquelética, a qual surge em regiões anatômicas diversas, em vários graus de intensidade. A dor musculoesquelética é apontada, em vários estudos que envolveram professores, como um relevante problema de saúde e as doenças decorrentes de agravos ao sistema musculoesquelético, é ainda uma das principais responsáveis por grande parte dos gastos com tratamento de saúde e indenizações, sendo a segunda causa de afastamento do trabalho no Brasil. ${ }^{11,15-17}$

Inúmeros aspectos da qualidade de vida de um indivíduo são importantes na manutenção da sua saúde emocional e os resultados desses desequilíbrios podem provocar uma diminuição social e psicológica dos docentes. 0 adoecimento psíquico forma um problema de expressiva relevância entre esses profissionais, tanto no que diz respeito às referências de sintomas de cansaço mental, nervosismo, quanto na identificação de transtornos mentais comuns. ${ }^{10,18}$

Os profissionais da educação quando apresentam baixa autoestima, ligada ao sentimento de pessimismo, fracasso, culpa e depressão, que podem surgir com déficit da qualidade de vida, torna receoso o sucesso de ações educacionais. São sentimentos que conduzem a atitudes de indecisão e irritabilidade, e podem prejudicar o processo educacional. Quando a qualidade de vida do professor é afetada devido as condições de trabalho, os efeitos se estendem ao ambiente de trabalho, podendo afetar ainda os outros sujeitos e até o resultado do seu próprio trabalho. ${ }^{6}$

Nesse contexto, o objetivo desse estudo é avaliar a qualidade de vida de professores do ensino fundamental de uma escola da rede pública brasileira, da cidade de Campina Grande/PB.

\section{MATERIAIS E MÉTODOS}

Trata-se de um estudo epidemiológico de corte transversal, de natureza descritiva e abordagem quantitativa, realizada em uma Escola Municipal de Ensino Fundamental, na cidade de Campina Grande/PB, no Brasil.

Seguindo os princípios éticos, esse estudo foi previamente submetido e aprovado pelo Comitê de Ética em Pesquisa (CEP) da UEPB, sob vigência da Resolução n466/12, de 12 de dezembro de 2012 do Conselho Nacional de Saúde/MS, sob número de CAEE 37458914.3.0000.5187. Antes da coleta das informações, os participantes que desejaram participar foram convidados a assinar um termo de compromisso Livre e Esclarecido em respeito aos aspectos éticos relativos a pesquisa com sujeitos humanos, conforme a resolução No 196/96.

A amostra foi instituída por 23 docentes, compreendendo os professores da educação infantil (pré-escola), ensino fundamental I ( $1^{\text {a }}$ a $4^{\text {a }}$ série) e ensino fundamental II ( $5^{\text {a }}$ a $8^{\text {a }}$ série).

A coleta das informações do estudo, foi realizada através da técnica de entrevista, com base na aplicação de um questionário dividido em quatro partes: a primeira ao qual abordava questionamentos sociodemográficos, de trabalho e estado geral de saúde; a segunda abordando a qualidade de vida, com o Short Form-36. ${ }^{19}$ A terceira parte possui questionamentos sobre avaliação dos sintomas osteomusculares, através do Questionário Nórdico. ${ }^{20}$ Por fim, foi avaliada a presença de depressão através do Inventário de Depressão de Beck. ${ }^{21}$

$\mathrm{Na}$ primeira parte do questionário inclui-se os dados de identificação do entrevistado, seguido das informações do perfil geral de trabalho e sobre estado geral de saúde e hábitos sociais dos professores. 0 Short Form (SF) 36 do instrumento Medical Outcomes Study (MOS), é formado por 36 itens que abordam conceitos físicos e mentais englobados em oito domínios: Capacidade funcional, Aspectos físicos, Dor, Estado Geral de Saúde, Vitalidade, Aspectos Sociais, Aspectos Emocionais e Saúde Mental. 0 escore é de 0 a 100, em que 100 indicando a melhor qualidade de vida possivel e 0 a pior. $^{22,23}$

0 Questionário Nórdico utilizado foi validado e adaptado para a língua portuguesa. 0 referido questionário tem como objetivo verificar a presença dos sintomas osteomusculares na última semana, no último ano e afastamento do trabalho no último ano devido à ocorrência da sintomatologia dolorosa. ${ }^{24}$

No que se diz respeito à depressão, a variável foi avaliada pelo Inventário de Depressão de Beck (Beck Depression Inventory BDI), criado para avaliar a intensidade de depressão. É uma escala constituída por 21 grupos de quatro afirmações. Os resultados alcançados neste questionário correspondem ao somatório dos valores atribuídos a cada afirmação, podendo assumir resultados entre 0 e 63 , no sentido de maior depressão. ${ }^{21}$

As informações foram coletadas por meio de entrevista, com base na aplicação de questionários estruturados. Os dados obtidos foram tabulados em planilha eletrônica Excel (Office do Microsoft, versão 2007) e foram analisados através do pacote estatístico 
Statistical Package for Social Sciences (SPSS) versão 19.0 (IBM Corp.,Armonk, Estados Unidos).

Os dados numéricos estão expostos sob a forma de média e desvio padrão, e apresentados os valores mínimos e máximos. Os dados categóricos são apresentados sob a forma de frequências. Para confrontar os domínios da qualidade de vida com a prevalência de dor aguda e crônica, e afastamento nos últimos 12 meses, utilizouse o teste de Mann-Whitney. A correlação entre depressão e qualidade de vida foi verificada por meio do teste de correlação de Spearman. Em todas as análises foi considerado um intervalo de confiança de 95\% (IC95\%) e significância estatística de p<0,05.

\section{RESULTADOS}

A amostra foi composta por 23 professores, com idade entre 27 a 66 anos, sendo a maioria do sexo feminino $(69,6 \%)$ e com cônjuge $(60,9 \%)$. Em relação ao nível de escolaridade, a maioria dos docentes apresentam apenas graduação $(56,5 \%)$. Considerando o estado geral de saúde e hábitos sociais, verificou-se, de acordo com a classificação do Índice de Massa Corporal (IMC), que 65,2\% dos docentes apresentam sobrepeso/obesidade. A frequência de fumantes atuais foi de $8,7 \%$. Sobre a prática de atividades físicas, $39,1 \%$ dos docentes que praticam algum tipo de atividade, a maioria a realiza até três vezes por semana (tabela 1).

Tabela 1.

Caracterização dos docentes entrevistados de acordo com os dados sociodemográficos, estado geral de saúde e hábitos sociais.

\begin{tabular}{|c|c|c|}
\hline Características Gerais & $\mathrm{N}$ & $\%$ \\
\hline \multicolumn{3}{|l|}{ Sexo } \\
\hline Masculino & 7 & 30,4 \\
\hline Feminino & 16 & 69,6 \\
\hline \multicolumn{3}{|l|}{ Grupo etário } \\
\hline Adulto jovem & 1 & 4,3 \\
\hline Adulto & 19 & 82,6 \\
\hline Idoso & 3 & 13,0 \\
\hline \multicolumn{3}{|l|}{ Estado Civil } \\
\hline Com Cônjuge & 14 & 60,9 \\
\hline Sem Cônjuge & 9 & 39,1 \\
\hline \multicolumn{3}{|l|}{ Escolaridade } \\
\hline Graduação & 13 & 56,5 \\
\hline Pós- graduação & 10 & 43,5 \\
\hline \multicolumn{3}{|l|}{ IMC } \\
\hline Eutrofia & 8 & 34,8 \\
\hline Sobrepeso/ Obesidade & 15 & 65,2 \\
\hline \multicolumn{3}{|l|}{ Tabagista } \\
\hline Sim & 2 & 8,7 \\
\hline Não & 21 & 91,3 \\
\hline \multicolumn{3}{|l|}{ Etilista } \\
\hline Sim & 5 & 21,7 \\
\hline Não & 18 & 78,3 \\
\hline \multicolumn{3}{|l|}{ Pratica atividade física } \\
\hline Sim & 9 & 39,1 \\
\hline Não & 14 & 60,9 \\
\hline \multicolumn{3}{|l|}{ Horas de sono } \\
\hline Até 6 horas & 10 & 43,5 \\
\hline Mais de 6 horas & 13 & 56,5 \\
\hline \multicolumn{3}{|l|}{ Prevalência de doenças } \\
\hline Sim & 6 & 26,1 \\
\hline Não & 17 & 73,9 \\
\hline
\end{tabular}

Fonte: Dados da Pesquisa, 2015.
Considerando as características relacionadas ao perfil geral do trabalho (tabela 2), observou-se que a maioria dos docentes caminha durante a execução de suas atividades $(34,8 \%)$. Foi visto que a maior parte dos professores entrevistados (87\%) costumam levar trabalho para casa, além disso, observou-se uma maior prevalência de professores $(65,2 \%)$ que trabalham em mais de uma escola, gerando uma dupla sobrecarga.

Considerando a distribuição da sintomatologia dolorosa músculo esquelética entre os professores entrevistados, foi visto um maior comprometimento de dor musculoesquelética referente aos últimos 7 dias nos membros inferiores $(34,8 \%)$. Ao considerar os sintomas relatados nos últimos 12 meses, o maior comprometimento $(47,8 \%)$ estava relacionado aos problemas na coluna (presença de dores, desconfortos ou dormência). No que diz respeito ao afastamento de suas atividades normais de trabalho nos últimos 12 meses, os docentes referiram como causa a ocorrência de problemas na região da coluna $(34,8 \%)$, nos membros superiores $(34,8 \%)$, e nos membros inferiores $(34,8 \%)$.

Conforme o Inventário de Depressão de Beck, a amostra apresentou uma média de 5,35 pontos $(\mathrm{DP} \pm 4,932)$, com valor mínimo de 0 e valor máximo de 20. Quanto à avaliação da qualidade de vida pelo SF-36, foram encontrados os valores de 84,38 para o domínio "Capacidades funcional" e 56,04 para "Estado geral de saúde", respectivamente, o melhor e o pior score médio, conforme visualizado na tabela 3 .

Tabela 2.

Caracterização dos docentes de acordo com os dados do perfil geral de trabalho.

\begin{tabular}{|c|c|c|}
\hline Perfil Geral de Trabalho & $\mathrm{N}$ & $\%$ \\
\hline \multicolumn{3}{|l|}{ Postura das Atividades } \\
\hline Sentado & 2 & 8,7 \\
\hline Em pé & 6 & 26,1 \\
\hline Sentado/ em pé & 7 & 30,4 \\
\hline Caminhando & 8 & 34,8 \\
\hline \multicolumn{3}{|l|}{ Levar trabalho para casa } \\
\hline Sim & 20 & 87,0 \\
\hline Não & 3 & 13,0 \\
\hline \multicolumn{3}{|l|}{ Horas de trabalho } \\
\hline Até 20 horas & 4 & 17,4 \\
\hline 21 a 30 horas & 13 & 56,5 \\
\hline Mais de 30 horas & 6 & 2,1 \\
\hline \multicolumn{3}{|l|}{ Tempo de docência } \\
\hline Menos de 5 anos & 1 & 4,3 \\
\hline De 5 a 10 anos & 1 & 4,3 \\
\hline 11 a 20 anos & 8 & 34,8 \\
\hline 21 a 30 anos & 9 & 39,1 \\
\hline Mais de 30 anos & 4 & 17,4 \\
\hline \multicolumn{3}{|l|}{ Turnos trabalhados } \\
\hline 1 Turno & 2 & 8,7 \\
\hline 2 Turnos & 14 & 60,9 \\
\hline 3 Turnos & 7 & 30,4 \\
\hline \multicolumn{3}{|c|}{ Usa transporte público para o trabalho } \\
\hline Sim & 9 & 39,1 \\
\hline Não & 14 & 60,9 \\
\hline \multicolumn{3}{|l|}{ Trabalha em outras escolas } \\
\hline Sim & 15 & 65,2 \\
\hline Não & 8 & 34,8 \\
\hline \multicolumn{3}{|l|}{ Tem outra profissão } \\
\hline Sim & 3 & 13,0 \\
\hline Não & 20 & 87,0 \\
\hline
\end{tabular}


Tabela 3.

Caracterização dos docentes de acordo com a qualidade de vida (SF-36).

$\begin{array}{lcccc}\text { Domínios do SF-36 } & \text { Média } & \text { DP }( \pm) & \begin{array}{c}\text { Valor } \\ \text { mínimo }\end{array} & \begin{array}{c}\text { Valor } \\ \text { máximo }\end{array} \\ \text { Capacidade Funcional } & 84,38 & 14,40 & 55,0 & 100,0 \\ \text { Limitação por aspectos físicos } & 79,34 & 26,81 & 25,0 & 100,0 \\ \text { Dor } & 62,45 & 23,69 & 10,0 & 100,0 \\ \text { Estado Geral de Saúde } & 56,04 & 11,93 & 37,0 & 82,0 \\ \text { Vitalidade } & 63,04 & 20,32 & 5,0 & 85,0 \\ \text { Aspectos Sociais } & 76,08 & 23,20 & 12,5 & 100,0 \\ \text { Limitação por aspectos emocionais } & 75,36 & 36,53 & 0 & 100,0 \\ \text { Saúde Mental } & 75,65 & 12,52 & 40,0 & 96,0\end{array}$

Fonte: Dados da Pesquisa, 2015.

No domínio "Estado geral de saúde" foi observado o menor valor médio, o que indica má avaliação da qualidade de vida deste item na população entrevistada, podendo este resultado ter repercussão direta na prevalência de morbidades entre os docentes, refletindo em afastamento da profissão da docência.

Quando comparada a qualidade de vida dos docentes com a prevalência de sintomatologia dolorosa e necessidade de afastamento devido a dor com os que não relataram dores, houve depreciação da qualidade de vida em 65,2\% do docentes que relataram dor aguda, 69,6\% daqueles que relataram prevalência de dor crônica e 56,5\% dos que se afastaram das atividades por conta da presença de dor.

Considerando os domínios de qualidade de vida entre os docentes com e sem dor aguda, foi observada uma diferença significativa apenas nos domínios "Limitações por aspectos físicos" $(p=0,02)$ e
"Estado geral de saúde" ( $\mathrm{p}=0,031)$, como pode ser observado na tabela 4.

Quando comparada a qualidade de vida dos professores entrevistados, considerando a presença de dor crônica e quanto aos docentes que relataram afastamento das suas atividades, observou-se uma diferença significativa no domínio "Limitação por aspectos físicos" em ambas situações ( $p=0,009 ; p=0,038$, respectivamente).

Correlacionando a depressão com a qualidade de vida dos professores, observou-se uma correlação negativa e moderada da depressão dos domínios "Limitações por aspectos físicos" ( $\mathrm{r}=-0,46$; $\mathrm{p}=0,026)$; "Dor" ( $\mathrm{r}=-0,58 ; \mathrm{p}=0,005)$; "Estado geral de saúde" $(\mathrm{r}=$ -0,57; $\mathrm{p}=0,010)$, "Vitalidade" $(\mathrm{r}=-0,65 ; \mathrm{p}=0,001)$, "Aspectos sociais" ( $r=-0,58 ; p=0,003)$. Observou-se, ainda, uma tendência para correlação entre depressão e o domínio Capacidade funcional, que apresentou $\mathrm{p}=0,051$, conforme tabela 5 .

Tabela 5.

Correlação entre Depressão e Qualidade de Vida dos docentes.

$\begin{array}{lcc} & \begin{array}{c}\text { Depressão } \\ r \text { Pearson }\end{array} & P \\ \text { Capacidade funcional } & -0,412 & 0,051 \\ \text { Limitação por aspectos físicos } & -0,463 & 0,026 \\ \text { Dor } & -0,579 & 0,005 \\ \text { Estado geral de saúde } & -0,526 & 0,010 \\ \text { Vitalidade } & -0,656 & 0,001 \\ \text { Aspectos sociais } & -0,584 & 0,003 \\ \text { Limitação por aspectos emocionais } & -0,315 & 0,143 \\ \text { Saúde Mental } & -0,265 & 0,221\end{array}$

$\mathrm{R}$ Pearson $=$ resultado do teste de correlação de Pearson; $p=$ nível de significância.

Tabela 4.

Comparação da qualidade de vida e sintomatologia de dor aguda e crônica e quanto ao afastamento do trabalho entre os docentes.

\begin{tabular}{|c|c|c|c|c|c|c|}
\hline Variáveis & $\mathrm{N}$ & Média & $\mathrm{DP}( \pm)$ & Mínimo & Máximo & $p$ \\
\hline \multicolumn{7}{|l|}{ Dor Aguda } \\
\hline Capacidade Funcional & 23 & 84,34 & 14,40 & 55,0 & 100,0 & 0,720 \\
\hline Limitação por aspectos físicos & 23 & 79,34 & 26,81 & 25,0 & 100,0 & 0,022 \\
\hline Dor & 22 & 62,45 & 23,69 & 10,0 & 100,0 & 0,068 \\
\hline Estado Geral de Saúde & 23 & 56,04 & 11,93 & 37,0 & 82,0 & 0,031 \\
\hline Vitalidade & 23 & 63,04 & 20,32 & 5,0 & 85,0 & 0,183 \\
\hline Aspectos Sociais & 23 & 76,08 & 23,20 & 12,5 & 100,0 & 0,205 \\
\hline Limitação por aspectos emocionais & 23 & 75,36 & 36,53 & 0,0 & 100,0 & 0,114 \\
\hline Saúde Mental & 23 & 75,65 & 12,52 & 40 & 96 & 0,556 \\
\hline \multicolumn{7}{|l|}{ Dor Crônica } \\
\hline Capacidade Funcional & 23 & 84,34 & 14,40 & 55,0 & 100,0 & 0,438 \\
\hline Limitação por aspectos físicos & 23 & 79,34 & 26,81 & 25,0 & 100,0 & 0,009 \\
\hline Dor & 22 & 62,45 & 23,69 & 10,0 & 100,0 & 0,095 \\
\hline Estado Geral de Saúde & 23 & 56,04 & 11,93 & 37,0 & 82,0 & 0,072 \\
\hline Vitalidade & 23 & 63,04 & 20,32 & 5,0 & 85,0 & 0,201 \\
\hline Aspectos Sociais & 23 & 76,08 & 23,20 & 12,5 & 100,0 & 0,254 \\
\hline Limitação por aspectos emocionais & 23 & 75,36 & 36,53 & 0,0 & 100,0 & 0,196 \\
\hline Saúde Mental & 23 & 75,65 & 12,52 & 40 & 96 & 0,710 \\
\hline \multicolumn{7}{|l|}{ Afastamento } \\
\hline Capacidade Funcional & 23 & 84,34 & 14,40 & 55,0 & 100,0 & 0,754 \\
\hline Limitação por aspectos físicos & 23 & 79,34 & 26,81 & 25,0 & 100,0 & 0,004 \\
\hline Dor & 22 & 62,45 & 23,69 & 10,0 & 100,0 & 0,006 \\
\hline Estado Geral de Saúde & 23 & 56,04 & 11,93 & 37,0 & 82,0 & 0,038 \\
\hline Vitalidade & 23 & 63,04 & 20,32 & 5,0 & 85,0 & 0,200 \\
\hline Aspectos Sociais & 23 & 76,08 & 23,20 & 12,5 & 100,0 & 0,320 \\
\hline Limitação por aspectos emocionais & 23 & 75,36 & 36,53 & 0,0 & 100,0 & 0,031 \\
\hline Saúde Mental & 23 & 75,65 & 12,529 & 40 & 96 & 0,055 \\
\hline
\end{tabular}

Fonte: Dados da Pesquisa, 2015. 


\section{DISCUSSÃO}

Segundo a Organização das Nações Unidas para Educação, Ciência e Cultura (Unesco) no Brasil 81,3\% dos professores eram do sexo feminino. Essa predominância de docentes do sexo feminino pode ser explicada através do processo histórico de inserção das mulheres no mercado de trabalho, sendo um campo de trabalho historicamente rotulado como extensão de trabalho doméstico, tornando-se uma alternativa para as mulheres se manterem no mercado de trabalho. ${ }^{8,25}$ Atualmente, esse campo profissional é ainda predominantemente desempenhado pelas mulheres. ${ }^{13,26}$

Considerando a faixa etária e a situação conjugal entre os docentes entrevistados observa-se que o presente estudo corrobora com outros estudos realizados com professores do ensino fundamental e médio. ${ }^{26,27}$ Tal semelhança também foi encontrada quanto às horas de trabalhos. Nos estudos de Silva et $\mathrm{al}^{28}$ e Marqueze e Moreno ${ }^{29} \mathrm{a}$ maior parte dos docentes trabalham por 8 horas diárias, ou seja, por mais de um turno de trabalho, corroborando com os achados no presente estudo.

Sobre a prática de atividades físicas, foram encontrados dados similares nos estudos de Delcor et $\mathrm{al}^{8}$, onde $38,9 \%$ dos professores do ensino pré-escolar até ensino médio a realizam, e na pesquisa de Reis et al. ${ }^{10}$ somente $30,8 \%$ dos docentes mencionaram práticas regulares de atividade física. Leandro ${ }^{30}$ afirma que quanto maior a pratica de atividade física realizada pelo professor, maior a capacidade nocioceptiva desse indivíduo. Dos 39,1\% dos docentes que praticam algum tipo de atividade, a maioria a realiza até três vezes por semana, porém, não atingindo a quantidade mínima recomendada pelos órgãos de saúde, dados que corroboram com os achados no estudo de Silva e Silva. ${ }^{26}$

No atual estudo verificou-se que há um maior relato de dor musculoesquelética em membros inferiores, considerando os 7 dias antecedentes à avaliação. É possivel observar semelhança com o estudo realizado por Cardoso et $\mathrm{al}^{31}$, com 4.496 professores do ensino fundamental na cidade de Salvador, BA. A tendência do docente permanecer por muito tempo na posição ortostática, durante a jornada de trabalho, ou caminhando para a realização das suas atividades, somando-se à necessidade de deslocar-se de uma escola para outra, algumas vezes por meio de caminhadas, mesmo que a maioria dos docentes do estudo não dependa de transporte público para ir à escola, são fatores que, quando combinados, segundo o estudo de Ribeiro $^{32}$ podem cooperar para sobrecarga dos membros inferiores, refletidos diariamente em forma de dor.

No estudo de Branco et $\mathrm{al}^{13}$, dados encontrados corroboraram com o presente estudo, onde demonstram uma prevalência geral de 89,7\% de sintomas osteomusculares em professores do ensino fundamental, ocorridos nos últimos 12 meses, com destaque para os altos índices de queixas de dores na coluna.

Sabe-se que a dor muscular tem se tornado um importante problema de saúde pública, provocando altos as custos sociais e econômicos, associando-se à depreciação da qualidade de vida da população estudada. Esses dados reforçam a hipótese de que o docente do ensino fundamental está submetido as condições de trabalho que favorecem o surgimento ou a manutenção da dor musculoesquelética, onde alguns mobiliários inadequados, desenvolvem posições desfavoráveis ao sistema musculoesquelético. ${ }^{32,33}$ Ribeiro et $\mathrm{al}^{32}$, observaram associação estatística entre ter turma única, trabalhar em dois ou mais turnos, carga horária semanal de 40 horas e não ter outra atividade remunerada, além da docência, com a prevalência de dores na coluna.
Em um estudo realizado com 320 professores do ensino fundamental de escolas públicas e privadas na zona urbana de Pelotas no que se refere à capacidade funcional, 36,6\% não conseguiram realizar suas atividades normais nos últimos doze meses devido à presença dos sintomas de dor e desconforto em pelo menos uma região anteriormente citada. ${ }^{13}$

Considerando a ocorrência de depressão, Strieder $^{34}$ observou em seu estudo, que há $29,79 \%$ dos professores apresentaram algum indicativo de depressão. Além disso, segundo Pereira et $\mathrm{al}^{35}$, as altas cargas horárias, número excessivo de alunos e turmas, pouco tempo para preparo das aulas, também esteve associado à presença de estresse relacionado ao trabalho. Por outro lado, observa-se que a capacidade funcional está relacionada à aptidão física, ou seja, a capacidade de realização de atividades diárias de um indivíduo, que tem de resistir e superar desafios e o estresse.

As características do desempenho desse profissional são marcadas por uma intensa jornada de trabalho. Uma explicação possivel para esses resultados pode ser a dupla jornada, aumentando assim a jornada diária de atividades e diminuindo o tempo para repouso, lazer e a necessária reposição de energia que, a longo prazo, pode originar diversas patologias musculoesqueléticas, muitas vezes relacionadas a quadros álgicos intensos, podendo acarretar em absenteísmos. Em estudos semelhantes um considerável percentual de docentes relataram não poder realizar atividades de trabalho e atividades domésticas, devido à alguma sintomatologia dolorosa, provocando repercussões sobre a qualidade de vida dos docentes. ${ }^{12,32}$

$\mathrm{Na}$ profissão docente, é inevitável o contato permanente com terceiros, o que se revela um constante desafio, o que alguns estudos afirmam ser um fator desencadeante responsável por desencadear medos e ameaças tanto no campo profissional como no campo pessoal, correlacionando-se com a presença de depressão. ${ }^{36}$ Segundo o estudo de Gomes e Quintão ${ }^{37}$, os professores que tem mais tempo de serviço, lecionavam em níveis de ensino inferiores, com carga letiva semanal superior e vínculo profissional mais definitivo, revelavam mais depressão. Em seu estudo Berber, Kupek e Berber ${ }^{38}$, a depressão mostrou-se responsável pela queda estatisticamente significativa dos escores de qualidade de vida.

Esta pesquisa possibilitou conhecer a qualidade de vida dos professores do ensino fundamental da escola em questão, e ainda a influência da sintomatologia de dor musculoesquelética e sintomatologia depressiva. Constatou-se que dentre os dados demográficos, as prevalências encontradas corroboram com a literatura quanto a depreciação da qualidade de vida da população estudada. Devido aos baixos salários os professores do ensino fundamental dobram a sua carga horária de trabalho, ou se submetem a outra profissão que não seja à docência, além de estarem diariamente expostos a fatores externos e internos, modificáveis ou não, que favorecem com o surgimento acarretando em um índice elevado de sintomatologias osteomusculares ou depressivas.

Ao identificar fatores ocupacionais que levam a depreciação da qualidade de vida, associados à ocorrência de dor musculoesquelética e sintomatologia dolorosa, é fortalecida a necessidade da adoção de medidas de prevenção aos agravos do sistema musculoesquelético, com o intuito de diminuir os afastamentos do trabalho e aposentadorias precoces, assim como melhorar a qualidade de vida e de trabalho do docente.

É de fundamental importância que sejam criadas discussões sobre educação e promoção de saúde no ambiente escolar, devendo evoluir para a operacionalização de programas que possibilitem o aumento da qualidade de vida dos professores. Também poderia ser observado 
o investimento para a presença de fisioterapeutas direcionados a trabalhos dentro da escola, viabilizando não apenas o desenvolvimento de atividades de educação postural junto aos escolares, como também um trabalho de prevenção e melhoria de qualidade de vida no trabalho dos docentes. Sugere-se a realização de novos estudos nessa população com a finalidade de investigar a associação entre as cargas de trabalho e determinados agravos saúde.
Estudo realizado na Universidade Estadual da Paraíba (UEPB), Campina Grande, PB, Brasil.

Financiamento próprio. Não há conflitos de interesse.

\section{REFERENCIAS}

1. Instituto Nacional de Estudos e Pesquisas Educacionais Anisio Teixeira (INEP) [on line]. Brasilia: INEP; 2013 [acesso 15 ago 2014]. Disponivel em: http://portal. inep.gov.br/.

2. Santomé JT. A construção da escolar pública como instituição democrática: Poder e participação da comunidade. Currículo sem Fronteiras. 2001; 1(1):51-80.

3. Limongi-França AC. Práticas de recursos humanos: conceitos, ferramentas e procedimentos. São Paulo: Atlas; 2007.

4. Francelino SM. As transformações do mundo do trabalho e a atividade docente. In: Leão, I.B. Educação e psicologia: reflexões a partir da teoria sócio-histórica. Campo Grande: UFMS; 2003. p.121-144.

5. Ministério do Trabalho e Emprego-Secretaria de Políticas Públicas do Emprego. (TEM-SPPE). Classificação Brasileira das Ocupações: CBO. Brasilia: MTE; 2002.

6. Mendes MLM. Condições de trabalho e saúde docente. Rio de Janeiro: Universidade Estadual do Rio de Janeiro; 2006.

7. Araújo TM, Silvany AM, Reis EJFB, Dutra FRD, Azi GR, Alves RL. Trabalho docente e sofrimento psíquico: um estudo entre professores de escolas particulares de Salvador. Ver FAEEBA. 2003; 12:485-495.

8. Delcor NS, Araújo TM, Reis EJFB, Porto LA, Carvalho FM, Silva MO, et al. Condições de trabalho e saúde dos professores da rede particular de ensino de Vitória da Conquista, Bahia, Brasil. Cad Saúde Pública. 2004; 20(1):187-196.

9. Reis EJFB, Carvalho FM, Araújo TM, Porto LA, Neto AMS. Trabalho e distúrbios psíquicos em professores da rede municipal de Vitória da Conquista, Bahia, Brasil. Cad Saúde Pública 2005; 21(5):1480-1490.

10. Reis EFB, Carvalho FM, Araújo TM, Porto LA, Neto AMS. Docência e exaustão emocional. Educ Soc (Campinas). 2006; 27(94):251-275.

11. Porto LA, Carvalho FM, Oliveira NF, Neto MAS, Araújo TM, Reis EJFB, Delcor NS. Associação entre distúrbios psíquicos e aspectos psicossociais o trabalho de professores. Rev Saúde Pública. 2006; 40(5):818-826.

12. Fernandes MH, da Rocha VM, Roncalli, Costa-Oliveira AG. Fatores associados à prevalência de sintomas osteomusculares em professores. Rev Salud Pública. 2009:11(2):256-267.

13. Branco CJ, Silva FG, Jansen K, Giusti PH. Prevalência de sintomas osteomusculares em professores de escolas públicas e privadas do ensino fundamental. Fisioter Mov. 2011; 24(2):307-314

14. Envasti J, Kivimäki $M$, Kawachi I, Subramanian SV, Pentti J, Oksanen T, Puusniekka $R$, et al. School environment as predictor of teacher sick leave: datalinked prospective cohort study. BMC Public Health. 2012;12:770.

15. Walsh IAP, Corral S, Franco RN, Canetti EEF, Alem MER, Coury HJCG. Capacidade para o trabalho em individuos com lesões músculo-esqueléticas crônicas. Rev Saúde Pública. 2004; 38(2):149-56.

16. Picoloto $D$, Silveira E. Prevalência de Sintomas Osteomusculares e Fatores Associados em Trabalhadores de uma Indústria Metalúrgica de Canoas, RS. Ciênc Saúde Coletiva. 2008; 13(2):507-516.

17. Mascarenhas CHM, Miranda PS. Sintomas de distúrbios osteomusculares relacionados ao exercício da assistência fisioterapêutica. Consciêntiae Saúde 2010, 9(3): 476-485

18. Libâneo JC. Adeus Professor, Adeus Professora? Novas exigências educacionais e profissão docente. 9a Ed. São Paulo: Cortez; 2006.

19. Ware JE. Sherbourne CD: The MOS 36 Item Short-Form Healt Survey (SF-36). I. Conceptual framework and item selection. Med Care. 1992; 30:473-483.

20. Mesquita CC, Ribeiro JC, Moreira P. Portuguese version of the standardized Nordic musculoskeletal questionnaire: cross cultural and reliability. J Public Health. 2010; 18(5):461-466.
21. Beck AT, Ward $C H$, Miendelson $M$, Mock J, Erbauch J. An inventory for measuring depression. Arch Gen Psychiatr. 1961; 4:53-63.

22. Martinez JE, MB Ferraz, Sato EL, Atra E. Avaliação da qualidade de vida de pacientes com fibromialgia através do "Medical Outcome Survey 36 Item Short- Form Study". Rev Bras Reumatol. 1999; 39(6):312-316.

23. Ciconelli RM, Ferraz MB, Santos W, Meinão I, Quaresma MR. Tradução para a língua portuguesa e validação do questionário genérico de avaliação de qualidade de vida SF-36 (Brasil SF-36). Rev Bras Reumatol. 1999;39(3):143-150.

24. Hernandez JAE, Neto FXV, Oliveira TC, Rodrigues AA, Neto CHE, Voser RC. Validação de construto do instrumento perfil do estilo de vida individual. Arq Mov. 2007; 3(1):3-17.

25. Belluci NP. Estranhamento; Alienação e Discriminação de Gênero: 0 Trabalho Da Mulher Professora. V Encontro Brasileiro de Educação e Marxismo. Florianópolis: UFSC; 2011.

26. Silva CM, Silva GL. Condições de trabalho e saúde de professores pré-escolares da rede pública de ensino de Pelotas, RS, Brasil. Ciênc Saúde Coletiva. 2013; 18(11):3137-3146.

27. Coelho TC, Neto $M$, Matos MA. Prevalência da sindrome do ombro doloroso (SOD) e sua influência na qualidade de vida em professores de uma instituição privada de nível superior na cidade de Lauro de Freitas, Bahia. Rev Baiana Saúde Pública 2010; 34(supl. 1):19-29.

28. Silva NEM, Figueirêdo DS, Freitas CES, Araújo TM, Paranhos IS. Trabalho docente em uma instituição de ensino superior da Bahia. VI Seminário da REDESTRADO-Regulação Educacional e trabalho Docente; 2006 Nov 6-7; Rio de Janeiro, Brasil. Rio de Janeiro: UERJ; 2006.

29. Marqueze EC, Moreno CRC. Satisfação no trabalho e capacidade para o trabalho entre docentes universitários. Psicol Estud. 2009; 14(1):75-82.

30. Leandro SX. Qualidade de vida e sintomatologia dolorosa musculoesquelética entre fisioterapeutas docentes de IES de Campina Grande, PB [dissertacao]. Campina Grande: Universidade Estadual da Paraiba; 2012. 49 p.

31. Cardoso JP, Ribeiro IQB, Araújo TA, Carvalho FM, Reis EJFB. Prevalência de dor musculoesquelética em professores. Rev Bras Epidemiol. 2009; 12(4): 604-614.

32. Ribeiro IQB, Araújo TM, Carvalho FM, Porto LA, Reis EJFB. Fatores ocupacionais associados à dor musculoesquelética em professores. Rev Baiana Saúde Pública. 2011; 35(1):42-64.

33. Chiu $\Pi$, Lam PK. The prevalence of and risk factors for neck pain and upper limb pain among secondary school teachers in Hong Kong. J Occup Rehabil. 2007; 17(1): 19-32

34. Strieder R. Depressão e ansiedade em profissionais da educação das regiões da Amerios e da AMEOSC. Roteiro. 2009; 34(2):243-268.

35. Pereira EF, Teixeira CL, Pelegrini A, Meyer C, Andrade RD, Lopes AS. Estresse Relacionado ao Trabalho em Professores de Educação Básica. Cienc trab. 2014. 16(51):206-210.

36. Capelo M, Pocinho M, Jesus SN. Stresse, estratégias de coping e autoeficácia em professores. Actas do I Congresso Luso-Brasileiro de Psicologia da Saúde; 2009 Fev 5-7; Algarve, Portugal. Algarve: UALG; 2009. p. 643-658.

37. Gomes APR, Quintão SR. Burnout, satisfação com a vida, depressão e carga horária em professores. Aná Psicológica. 2011;2(29):335-344.

38. Berber JSS, Kupek E, Berber SC. Prevalência de depressão e sua relação com a qualidade e vida em pacientes com sindrome a fibromialgia. Rev Bras Reumatol. 2005; 45(2):47-54. 